\title{
Applications of Micro-Raman Imaging in Biomedical Research
}

\author{
C. Otto, * C. J. de Grauw, J. J. Duindam, N. M. Sijtsema and J. Greve \\ University of Twente, Applied Optics Group and BioMedical Technology Institute, P.O. Box 217, 7500 AE Enschede, The \\ Netherlands
}

\begin{abstract}
Recent results are presented of the application of imaging micro-Raman spectrometers in cellular biophysics and biomedical research. Various micro-Raman spectrometers have been developed that are now routinely applied in these fields. Results are presented that were obtained with a linescan Raman microspectrometer and with a Raman imaging microscope. Applications of Raman linescan spectrometry concern the investigation of polytene chromosomes obtained from the salivary gland of Chironomus thummi thummi. The distribution of DNA and proteins was investigated in bands and interbands. In a second example of Raman linescan spectrometry, bone implants were investigated. These bone implants were coated with thin layers of materials that improve biocompatibility. The density, crystallinity and protein distribution can be investigated. Information from Raman imaging may help in selecting the proper materials for maximum biocompatibility. Raman imaging microscopy is used whenever twodimensional spatial information is required on the distribution of molecules or molecular components. Raman images are presented of filipine and phenylalanine in human eye-lenses in and around radial shades. Raman imaging is a particularly important tool for the study of the distribution of non-fluorescent drugs inside living cells. This is illustrated for the non-fluorescent drug cobalt octacarboxyphthalocyanine. (C) 1997 by John Wiley \& Sons, Ltd.
\end{abstract}

\section{INTRODUCTION}

The development of Raman microspectroscopy and Raman imaging has been reported. ${ }^{1,2}$ The potential for studies of single living cells has also been addressed. ${ }^{1}$ Apart from the development of lasers, other aspects particularly important in acquiring the required sensitivity in micro-raman imaging of biological systems are the advent of charge coupled devices (CCDs) as low-noise two-dimensional detectors and the exploitation of the potential of thin-layer techniques for steep-edged and high-contrast narrow-band and cut-off filters and the production of poly-chromators that have a maximized throughput and that are astigmatism corrected for high quality image transmission. The state-of-the-art equipment is now of such quality that no further major improvements in individual components can be expected. However, significant light losses still accumulate and even today's spectrometers detect only a fraction $^{3}(c a .15-35 \%)$ of all the Raman scattered light collected by the objective. This figure can be achieved after optimization for a selected wavenumber region. For a general-purpose instrument that can be used throughout the visible and near-infrared region, the absolute detection efficiency will be lower.

The development of extremely sensitive Raman spectrometers in our laboratory was driven by the desire to investigate, directly and non-invasively, living biological objects such as cells and chromosomes. Application of a standard Raman microspectrometer to a pelleted human chromosome ${ }^{4}$ sample resulted in a spectrum with a good signal-to-noise ratio. Bands were

* Author to whom correspondence should be addressed. assigned to the nucleic acid residues and to proteins. It was observed that DNA was mainly in the B-form. The spectrum could be interpreted in details in terms of the DNA to protein ratio and the A-T/G-C content. Shortly thereafter the Raman spectrum of a micrometer-sized single salmon sperm was obtained. ${ }^{5}$ The combination of confocal microscopic methods ${ }^{6}$ and Raman microspectrometry has been implemented in our laboratory ${ }^{3,7}$ and successfully applied to single chromosomes $^{8}$ and single living cells. ${ }^{9}$

The potential of molecular image formation on the basis of Raman microspectroscopy was recognized in the early 1970s. ${ }^{10}$ A number of instrumental layouts have been proposed for both linescan imaging ${ }^{11-13}$ and global imaging. ${ }^{14,15}$ In linescan imaging, the twodimensional camera contains spatial information in one direction and frequency information, in the form of the Raman spectrum, in the direction perpendicular to it. In global imaging a narrow band of wavenumbers, preferably with a bandwidth of $10 \mathrm{~cm}^{-1}$ or less, is selected from the total spectrum scattered by a sample and used to reconstruct an image of the object on the CCD camera.

In this paper, results are presented of Raman linescan imaging of bone implants and polytene chromosomes of Chironomus thummi thummi and of direct imaging Raman microscopy of eye-lens opacities and the distribution of the non-fluorescent drug cobalt octacarboxyphthalocyanine $\left[\mathrm{Co}(\mathrm{COO})_{8} \mathrm{Pc}\right]$ inside a $\mathrm{K} 562$ cell.

\section{EXPERIMENTAL}

The linescan Raman spectrometer is based on an instrument described previously. ${ }^{3}$ Red laser excitation can be 
selected between 630 and $690 \mathrm{~nm}$ in a continuous-wave DCM dye laser pumped by the all-lines-output of an argon ion laser. A scanning mirror is positioned in the excitation pathway before the dichroic mirror. The Raman emission is imaged on to a slit, in a plane conjugate with the object plane, before passing through a filter that suppresses the excitation wavelength ( $c a .660$ $\mathrm{nm}$ ). The slit has a width of $100 \mu \mathrm{m}$ in the spectral direction and $3 \mathrm{~mm}$ in the spatial direction. For the measurement on bone implants, a microscope objective (dry; Spindler and Hoyer) with $63 \times$ magnification and a numerical aperture of 0.8 was chosen. The dimensions of the linescan illumination are $0.5 \times 35 \mu \mathrm{m}$. For the measurements on polytene chromosomes a water immersion objective (Zeiss Plan Neofluor) with $63 \times$ magnification was used. The numerical aperture is 1.2. The size of the scanned area is $0.5 \times 25 \mu \mathrm{m}$.

The Raman imaging microscope has been described previously. ${ }^{15}$ The Raman band (bandwidth ca. $10 \mathrm{~cm}^{-1}$ ) in which the object is imaged is selected by the proper combination of the laser excitation wavelength and a transmission filter (Omega Optical).

The bone implant consisted of a titanium rod coated with a layer of crystalline hydroxyapatite. Hydroxyapatite (HAP) (CAM-Implants, Leiden, The Netherlands) was plasma sprayed on to pure titanium rods. The crystalline hydroxyapatite was then obtained by a thermal treatment of the amorphous sprayed coating. A smooth coating was obtained with a layer thickness ranging from $30-50 \mu \mathrm{m}$. The implants were inserted in goat legs and recovered after 6 weeks. The excised implants were dehydrated using ethanol and embedded in poly(methyl methacrylate). After extraction, thin disks were prepared from the cross-section of the implant. The sample was placed under the Raman microscope in such a way that only a small fraction of the linescan illuminated the titanium rod. No Raman signal could be obtained from this material. The laser power at the sample was $40 \mathrm{~mW}$. Spectra were acquired in $300 \mathrm{~s}$. An excellent signal-to-noise ratio was achieved in this time.

Polytene chromosomes were extracted from the salivary gland of fourth instar larvea of Chironomus thummi thummi, essentially following a procedure described previously. ${ }^{16}$ The physiologically isolated chromosomes were placed on a quartz microscope slide coated with poly(L-lysine) and immersed in Ringer's buffer. The chromosomes were not treated by any fixation procedure. A proper section of a polytene chromosome was selected with the help of the bright field illumination of the microscope. The size of the scan area was $0.5 \times 30$ $\mu \mathrm{m}$. The laser power at the sample was $24 \mathrm{~mW}$.

Eye-lenses were fixed using a procedure described previously. ${ }^{17}$ Using three parallel razor blades, slices with a thickness of $700 \mu \mathrm{m}$ were obtained from eyelenses containing cataractous spots. The eye-lenses were cut along the optical axis. The slices were incubated for $4 \mathrm{~h}$ at $38^{\circ} \mathrm{C}$ in the dark in a filipin solution (concentration $10^{-4} \mathrm{M}$ ) containing cacodylate to prevent bacterial growth. The filipin is considered as a Raman label. It binds to unesterified 3- $\beta$-hydroxysterols with an apolar chain at $C_{17}$ in a $1: 1$ sterol to filipin ratio. ${ }^{18,19}$ The radial shades could be observed with the white light utility in the base of the microscope. The laser wavelength was $647 \mathrm{~nm}$ from a krypton ion laser. The power at the sample was $1 \mathrm{~mW} \mu \mathrm{m}^{-2}$

Human leukemic erythroblasts (K562 cells) were used to investigate the distribution in cells of the nonfluorescent drug cobalt octacarboxyphthalocyanine, which was synthesized at the Moscow Institute of Organic Intermediates and Dyes. The cells were incubated at $37^{\circ} \mathrm{C}$ for $\mathrm{ca} .12 \mathrm{~h}$ in cellular growth medium containing a $10^{-4} \mathrm{M}$ concentration of the drug. The cells were transferred to a poly(L-lysine)-coated quartz cover-glass after washing with drug-free medium. The Raman image was obtained with a laser excitation wavelength at $660 \mathrm{~nm}$. The background image was obtained with a laser excitation of $658 \mathrm{~nm}$, in which case the narrow bandpass filter passed light with a Raman shift of $1600 \mathrm{~cm}^{-1}$.

\section{RESULTS}

\section{Bone implants}

In Fig. 1 a white light image of a metal bone implant coated with hydroxyapatite (HAP) is shown. The HAP coating on the titanium rod (left-hand side) can clearly be distinguished of the bone material (right-hand side). The titanium is not Raman active. The Raman linescan is therefore chosen to start in the bone and end in the HAP. In Fig. 2 the two-dimensional camera read-out after a measuring time of $300 \mathrm{~s}$ is shown. The phosphate vibration at $960 \mathrm{~cm}^{-1}$ and vibrations around 1450 $\mathrm{cm}^{-1}$ are characteristic of the bone material. For HAP an intense band at $960 \mathrm{~cm}^{-1}$ and bands at 1048 and $592 \mathrm{~cm}^{-1}$ are observed. In Fig. 3 the integrated intensity of the $960 \mathrm{~cm}^{-1}$ band of the totally symmetric

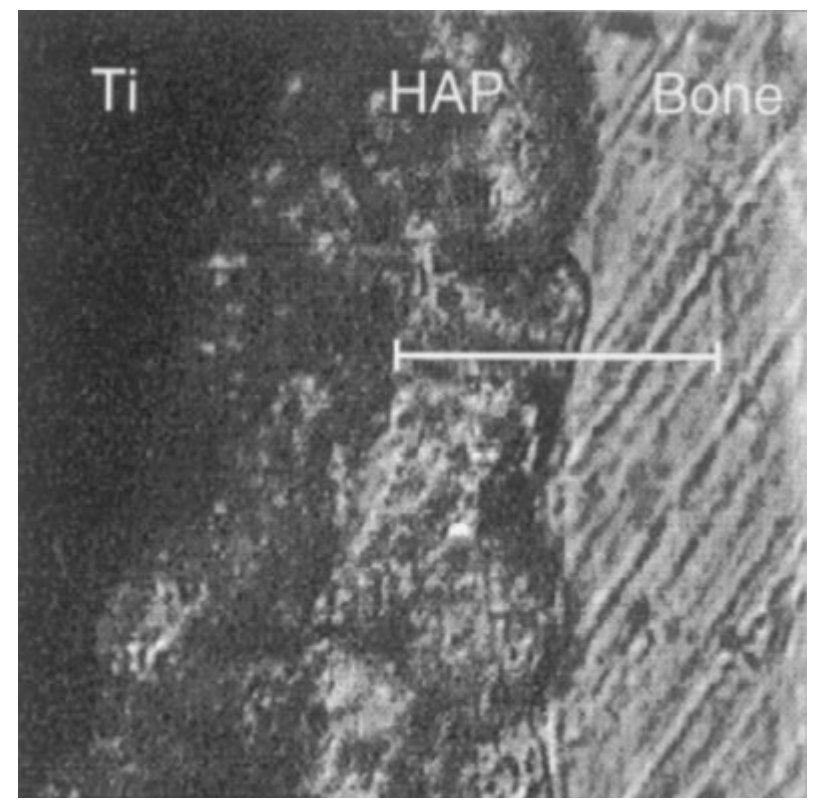

Figure 1. White light image of a section of a bone implant. The bone implant consists of a titanium rod coated with a layer of crystalline hydroxyapatite with a thickness of $c a .40 \mu \mathrm{m}$. On the righthand side the bone is situated. The white bar shows the area illuminated in the linescan. The scan amplitude is $30 \mu \mathrm{m}$. The linescan is positioned such as to cross the border between crystalline hydroxyapatite and the bone. 


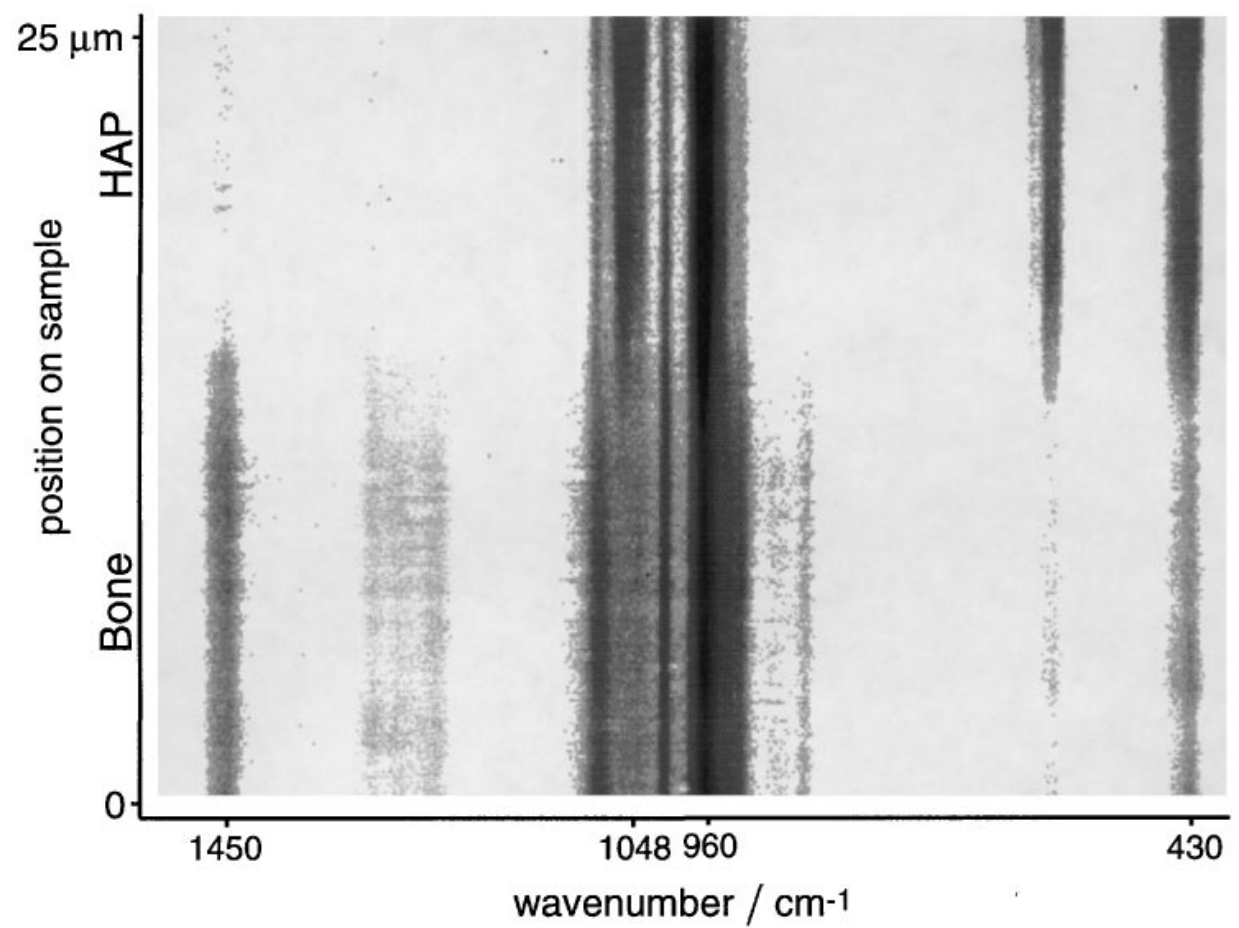

Figure 2. Raman linescan of a bone implant. Signal on the two-dimensional CCD array after a measurement time of $300 \mathrm{~s}$ and using a laser power of $40 \mathrm{~mW}$. The gray scale varies from 200 counts (white) to 2000 counts (black). Along the vertical direction spatial information is presented and along the horizontal direction the spectral information is presented. The most intense band in the spectrum is the symmetric phosphate-oxygen stretch vibration at $960 \mathrm{~cm}^{-1}$ from hydroxyapatite. This material is present both in the bone material (from $c a$. 0-8 $\mu \mathrm{m}$ ) and in the hydroxyapatite coating (from ca. 8-25 $\mu \mathrm{m}$ ), but with different intensities.

stretching mode of the phosphate group is displayed as a function of the position for three different linescans. The intensities are scaled from $0-100 \%$ at an arbitrary position. A sudden transition from the crystalline HAP of the coating to the weak HAP signal of the bone material itself is observed.

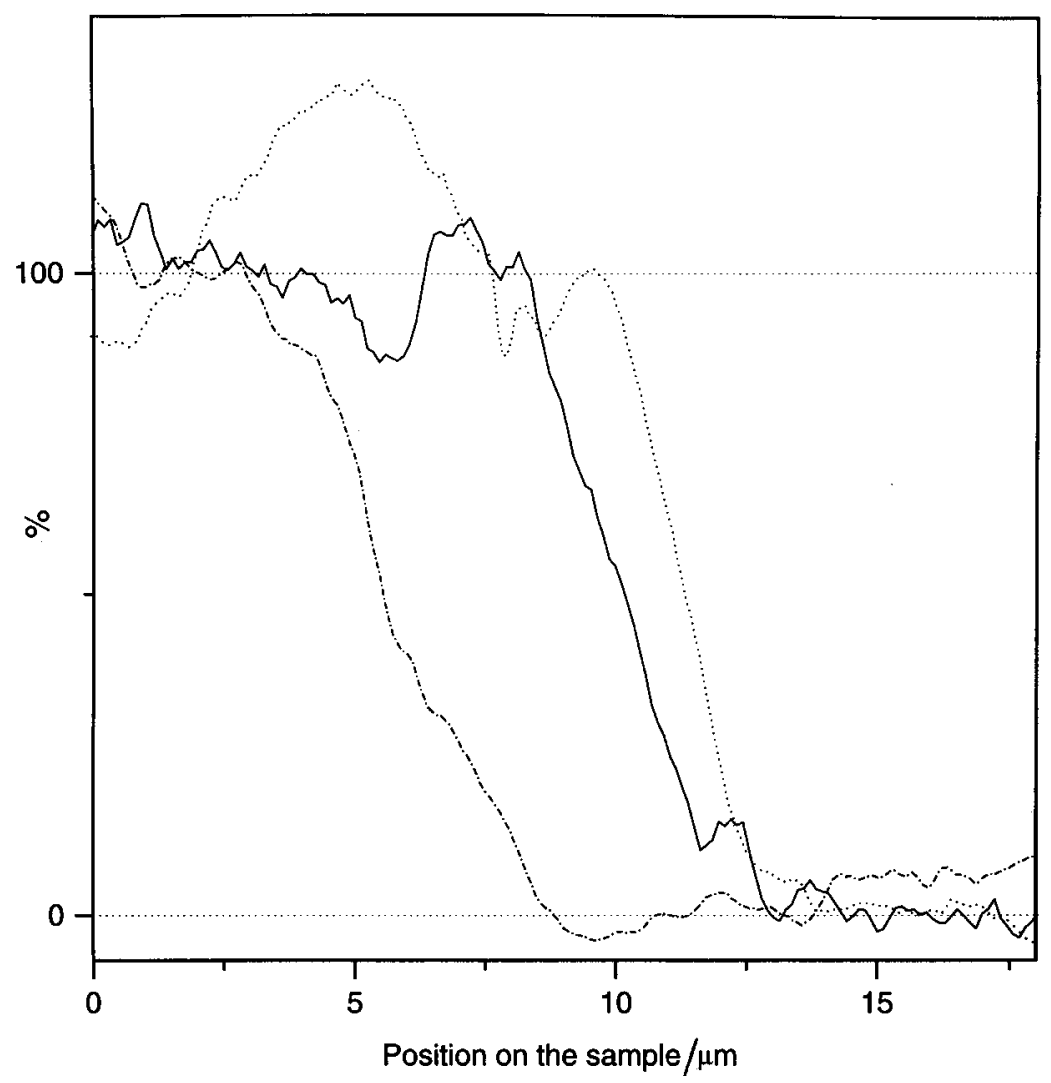

Figure 3. Intensity profiles of the symmetric phosphate-oxygen stretch vibration at $960 \mathrm{~cm}^{-1}$ from hydroxyapatite for three different linescans. The profile is scaled from $0 \%$ (intensity in the bone material) to $100 \%$ (intensity at some position in the hydroxyapatite coating). 


\section{Polytene chromosomes}

Figure 4 shows the white light image of a physiologically prepared polytene chromosome from Chironomus thummi thummi. The image of the two-dimensional camera read-out is portrayed next to the white light picture. The measurement time was $1800 \mathrm{~s}$. The linescan of the laser beam is shown as a white bar in the white light picture. The amplitude of the linescan covered 30 $\mu \mathrm{m}$ of the chromosome. The turning points of the linescan are discarded because the mirror velocity is not constant. Polytene chromosomes consist of alternating patterns of bands and interbands. The bands, showing up as dark areas in the white light picture, are identified by a higher Raman scattering activity. Hence the intensity distribution of the Raman scattering reveals that the dark horizontal bands in the Raman image coincide with interbands in the sample. This indicates that the number of Raman scatterers per $\mu \mathrm{m}^{3}$ in interbands is smaller than that in the bands. Taking a suitable average over rows of spectra in one band and one interband region gives good-quality Raman spectra. In Fig. 5 a band and interband spectrum are presented as extracted from the linescan image in Fig. 4.

\section{Cataractous spots in human eye-lenses}

A comparison of the Raman spectra from a large number of radial shades with healthy eye-lens tissue indicates that in the radial shades a considerable change has occurred in molecular composition and amino acid composition. ${ }^{20}$ In general, the radial shades contained a decreased amount of the aromatic amino-acid residues

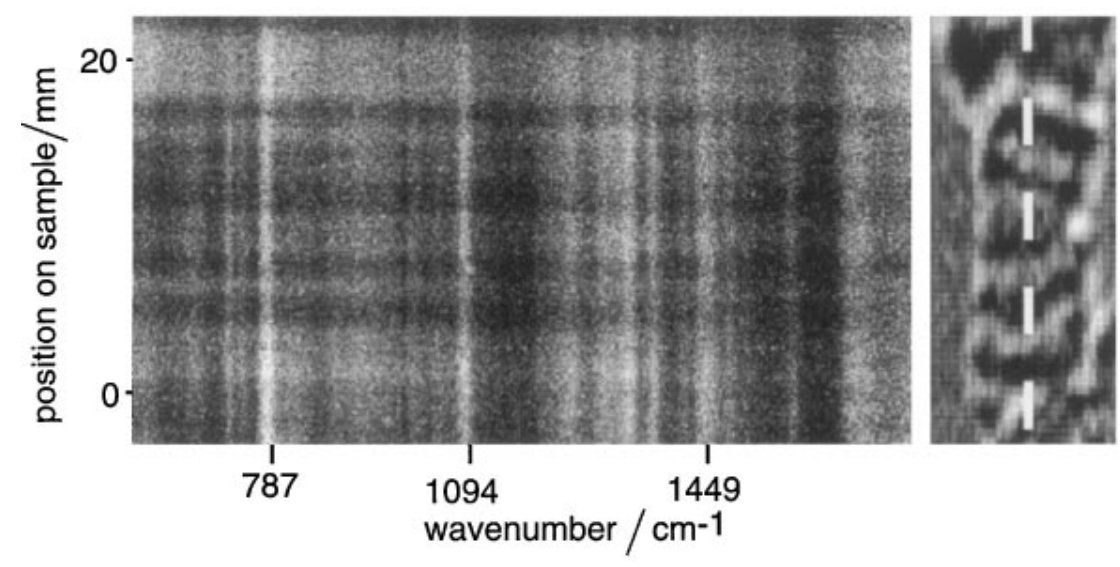

Figure 4. Raman linescan of a polytene chromosome from Chironomus thummi thummi. Signal on the two-dimensional CCD array after a measurement time of $1800 \mathrm{~s}$ and using a laser power of $48 \mathrm{~mW}$. Along the vertical direction spatial information is presented and along the horizontal direction the spectral information is presented. A section of $50 \mu \mathrm{m}$ of the total scan area of $60 \mu \mathrm{m}$ is shown. Next to the Raman linescan image the white light image of the selected part of the chromosome is shown. It can be observed directly that bright bands in the Raman linescan coincide with the dark bands in the white light image. The intensity of the Raman scattering from band areas is therefore larger than from interbands.

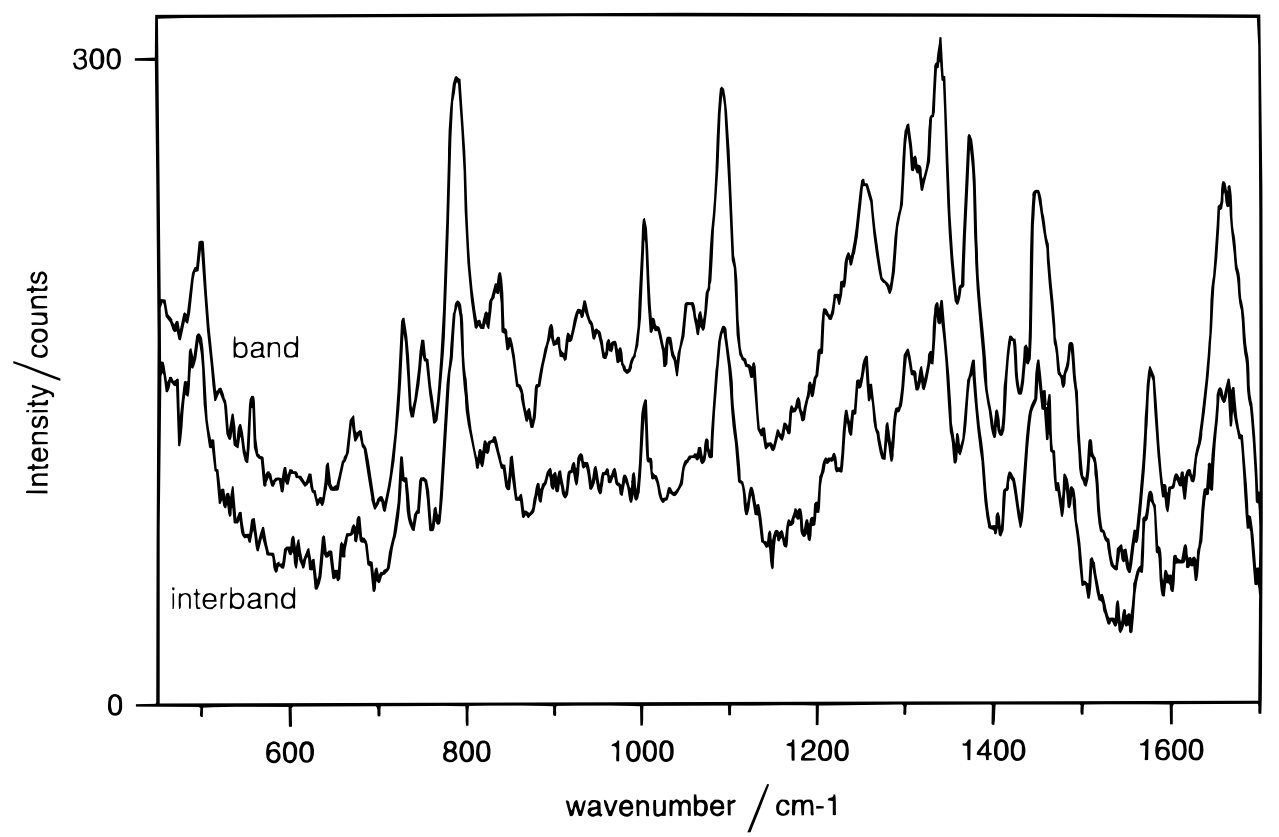

Figure 5. Sum of spectra of channels, containing the spectrum of a single band, compared with that of the neighboring interband. Typical DNA/protein spectra are obtained in both cases. The intensity in the Raman spectrum is larger in the band than in the interband. 


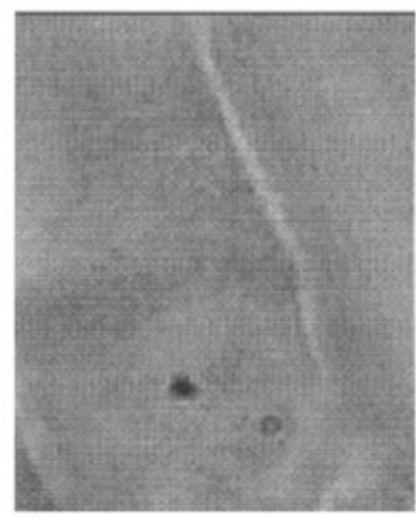

A
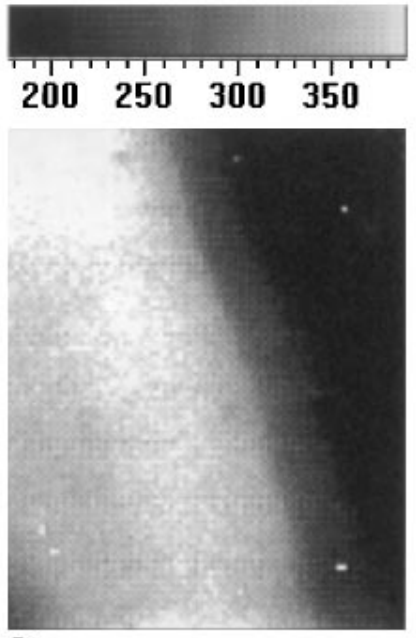

B
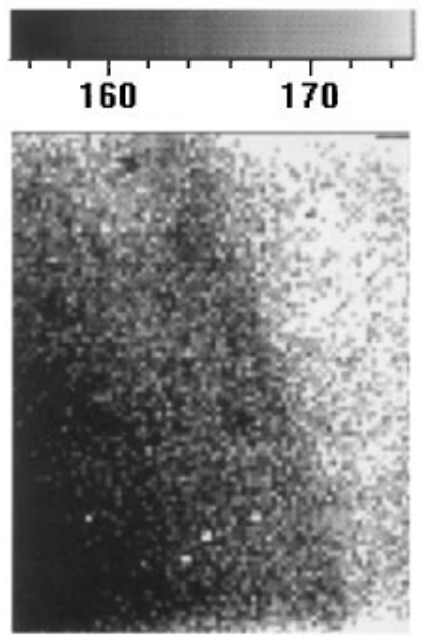

C

Figure 6. Global Raman imaging of radial shades in human eye lenses. (A) A white light image of a part of a radial shade. The boundary between the opacity and the healthy eye lens tissue can be discerned. (B) The Raman image at $1586 \mathrm{~cm}^{-1}$ at which wavenumber the ethylene chain vibration of filipine is observed. The extrinsic Raman label filipin binds stoichiometrically to cholesterol with an unesterified $3-\beta-O H$ group. The radial shade is clearly observed in white against the eye lens tissue in black. (C) The Raman image at $1004 \mathrm{~cm}^{-1}$ at which wavenumber the monosubstituted ring breathing mode of benzene is observed. The image with the intrinsic Raman label phenylalanine shows that the concentration of this aromatic amino acid has decreased in the opacity.

tryptophan, tyrosine and phenylalanine. Use of the Raman probe molecule filipin for cholesterol with unesterified $3-\beta-\mathrm{OH}$ groups indicates that these cholesterols have an increased concentration inside the cataractous spot. Initial experiments of such a kind were performed on a point-by-point basis. ${ }^{17}$ Raman imaging of the cataractous spots at various wavenumbers, characteristics of the various groups mentioned above, resulted in Fig. 6, where a small part of a slice of a human eye-lens was observed in (A) white light, (B) a filipine image and (C) a phenylalanine image. The contrast between the eye-lens tissue in the cataractous spot [left-hand side of Fig. 6(A)] and the healthy eye-lens tissue on the righthand side is small, but easily observable in white light microscopy. Raman imaging of the same area at the scattering wavelength of $1586 \mathrm{~cm}^{-1}$, which is coincident with the strongest vibration of filipine, gives a high contrast between the cataractous spot and the healthy tissue [Fig. 6(B)]. The high concentration of filipine in the cataractous spot equates to a high concentration of cholesterol with unesterified $3-\beta-\mathrm{OH}$ groups. Imaging the difference in the molecular composition on the basis

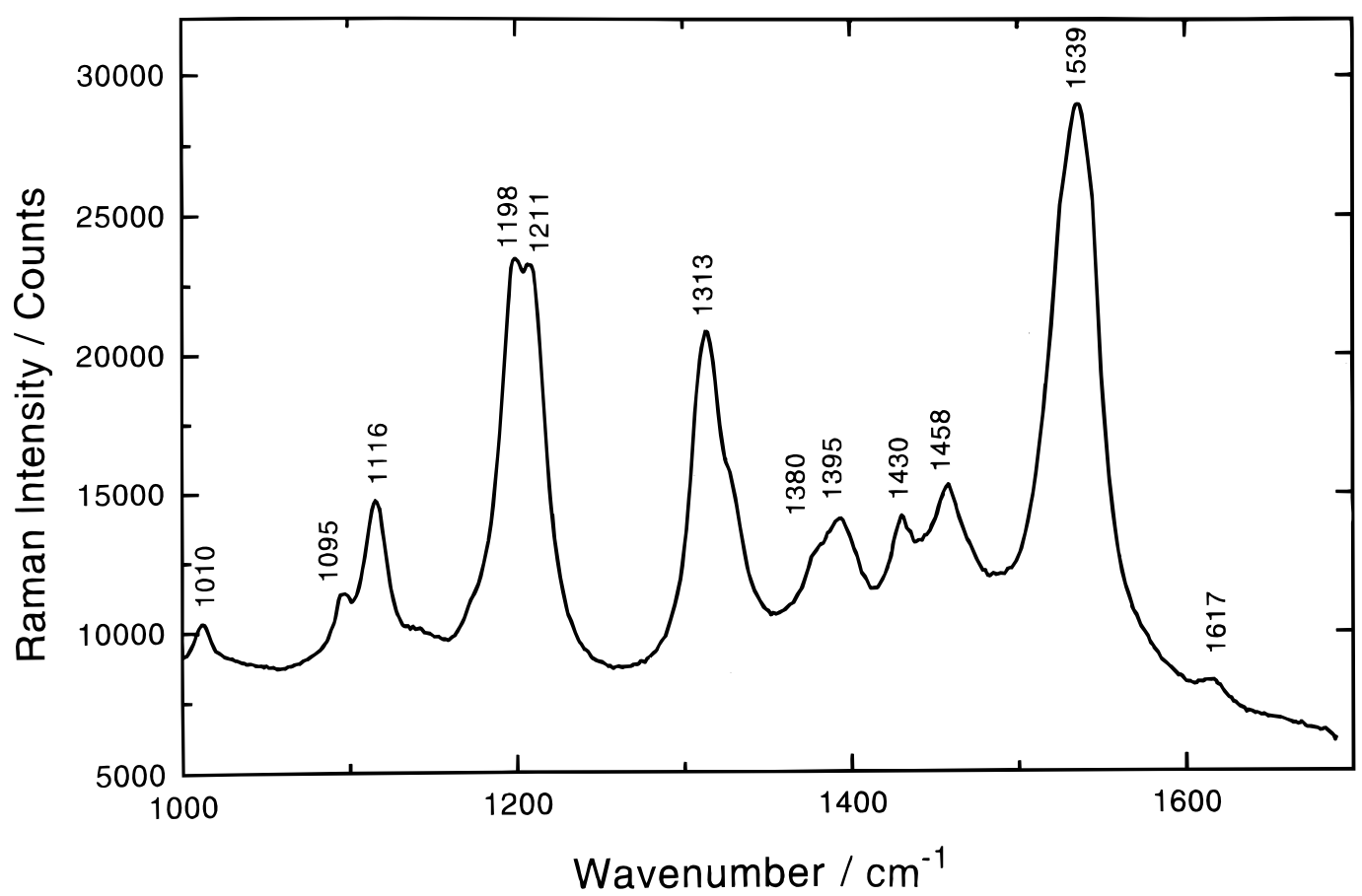

Figure 7. Raman spectrum of cobalt octacarboxyphthalocyanine from 1000-1650 $\mathrm{cm}^{-1}$. The strongest band at $1539 \mathrm{~cm}^{-1}$ is selected for imaging. 
of a characteristic band at $1004 \mathrm{~cm}^{-1}$ of the phenylalanine residue [Fig. $6(\mathrm{C})$ ] shows that this is indeed a characteristic of the material inside the radial shade and not of a fortuitously chosen position inside the cataractous spot.

\section{Cobalt octacarboxyphthalocyanine in single living cells}

Phthalocyanine derivatives are used in the photodynamic therapy of tumors. Selective accumulation in malignant cells is required, after which irradiation with light starts of the formation of cytotoxic compounds. The localization and distribution of the phthalocyanine molecules can be studied with fluorescence spectroscopy. Cobalt octacarboxyphthalocyanine, however, is not fluorescent. Raman imaging was used to study the distribution of this compound in K562 cells. Figure 7 shows the Raman spectrum from $1000-1650 \mathrm{~cm}^{-1}$. The strongest band in the spectrum is at $1539 \mathrm{~cm}^{-1}$ and is used for Raman imaging. Figure 8 presents (a) the white light image of a $\mathrm{K} 562$ cell incubated with $\mathrm{Co}(\mathrm{COO})_{8} \mathrm{Pc}$ together with (b) the Raman image obtained at 1539 $\mathrm{cm}^{-1}$.

\section{DISCUSSION}

Micro-Raman linescan spectrometry and micro-Raman global imaging have been shown to provide clear images of the molecular distribution in a number of biophysical objects. The power level used to achieve a relevant spatial resolution is $c a .1 \mathrm{~mW} \mu \mathrm{m}^{-2}$. The measuring time depends in detail on the magnitude and distribution of the Raman scattering activity in the sample. In the examples presented in this paper, the measurement time ranges from a few minutes for hydroxyapatite imaging in bone samples to $20-30 \mathrm{~min}$ for an image of the banding pattern in a giant chromosome of Chironomus thummi thummi. The examples clearly demonstrate the importance of an image of the molecular distribution in addition to the wavenumber information normally observed in a Raman spectrum. The lateral resolution in the Raman linescan spectrometer varies between 0.9 and $1.6 \mu \mathrm{m}$ and depends on the optical properties of the sample. This resolution is smaller than the diffraction-limited resolution. This results from the fact that only the incident laser light is scanned and the Raman scattered light is not. Any confocal aperture causes that the light cone that images sharply a point $(x, y)$ in some plane $Z$ will also collect light from a different plane $Z^{\prime}$ with $\left(x^{\prime}, y^{\prime}\right)$ in focus. This can be improved when not only the incident laser beam is scanned but also the resultant Raman scattered beam is synchronously scanned. The confocal aperture can in that case always be chosen as a circular aperture, resulting in decreased interplane cross-talk and higher three-dimensional spatial resolution.

\section{Bone implants}

The importance of the study of coated metal bone implants with Raman microspectroscopy lies in the fact that chemical and structural information are obtained at micrometer resolution. The structure and homogeneity of a coating, resulting from a plasma spray process, can be assessed both prior to and after implantation. The influence of living tissues on the coating materials can be studied. The use of a coating should lead to an increased affinity of natural bone to form bonds with the implant. It is clear from Fig. 2 that the spectrum of bone is different from that of the crystalline hydroxyapatite that was present as coating material. Especially bands in the high-wavenumber area around 1670 , around 1450, between 1300 and 1200 and between 950 and $800 \mathrm{~cm}^{-1}$ are present in bone but not in HAP. The bands can be ascribed to proteinic material (presumably collagen) and belong to, respectively, the amide $\mathrm{I}$ band, the $\mathrm{CH}_{2}$ and $\mathrm{CH}_{3}$ bending modes, the amide III band and the rocking modes in the aliphatic side-chains of the amino acids valine, leucine and lysine.

From the spectra in Fig. 2 it is apparent that large changes in the bandwidth occur in phosphate vibrations between bone and HAP. The bandwidths are much smaller in HAP than in the bone material. The bone material has a higher degree of amorphous structures. Furthermore, the presence of small amounts of negatively charged ions other than phosphate ions may lead to even larger variations in lattice constants, resulting in larger bandwidths. ${ }^{21}$ In Fig. 3, the integrated intensity of the $960 \mathrm{~cm}^{-1}$ band of the totally symmetric stretching mode of the phosphate group is displayed as a function of position for three different linescans. This picture reflects what can also be observed in Fig. 1, namely that the interface between HAP and bone is rather irregular. This irregularity extends in three dimensions. Figure 3 further reveals that large intensity fluctuations occur in the area where the HAP signal originates. This offers a direct interpretation of the dark and lighter parts in the HAP coating as observed under the white light illumination of the microscope in Fig. 1. The darker parts can be associated with higher concentrations of HAP. As the bandwidths of the $960 \mathrm{~cm}^{-1}$ Raman band do not depend on the position in HAP, variations in crystal structure and composition can be ruled out as an explanation.

\section{Polytene chromosomes}

The spatially resolved (vertical axis) Raman spectra (horizontal axis) in Fig. 4 are of good quality. It is concluded that excellent one-dimensional (spatially) Raman images can be obtained of complex biological systems with $c a .1 .5 \mathrm{~mW}$ of laser light per $\mu \mathrm{m}^{2}$. The intriguing question standing out from both the white light picture and the Raman picture in Fig. 4 is what the reason for the occurrence of the banding pattern is? The information in the Raman picture can be associated with the occurrence of the varies molecular species that can be expected, i.e. DNA, RNA, histone proteins and nonhistone proteins. Previously, it was shown ${ }^{22}$ that in fixed Chironomus thummi thummi chromosomes interbands had a decreased DNA content and also a decreased protein content with respect to the bands. Moreover, the Raman intensities of bands of DNA and bands of protein in different bands remained constant to within $5 \%$, whereas the intensities fluctuated from one interband to another. The physiologically prepared 
chromosome imaged in Fig. 4 does not reveal such a behaviour. It had not undergone any acid fixation that is responsible for protein distraction and DNA rupture. It is concluded from the Raman line imaging of freshly prepared chromosomes under physiological conditions that the interbands contain a lower density of both DNA and proteins as compared with the bands. The DNA to protein ratio is not dependent on the position in central sections of the chromosome.

\section{Cataractous spots in human eye-lenses}

The occurrence of cataractous spots in human eyelenses is an important health issue. Raman microspectroscopy is a powerful technique for obtaining information on the molecular composition of cataractous spots. In this particular study we show the first Raman images of cataractous spots. The images result from differences in the Raman spectrum of the cataractous spot with respect to the surrounding healthy eyelens tissue. The first Raman image resulted from a distribution of filipine and was recorded at a Raman shift of $1586 \mathrm{~cm}^{-1}$. Filipine can be considered as a Raman label for cholesterol with unesterified $3-\beta-\mathrm{OH}$ groups. Using this label it was shown, ${ }^{23}$ with optical resolution, that an age-related change occurs of the 3- $\beta$ hydroxycholesterol distribution along the optical axis of the lens. Here it is shown that filipin accumulates significantly more in the radial shades than in the surrounding healthy lens tissue. This can be related to the presence of large amounts of lipid molecular layers. The imaging directly shows that the distribution of filipine in the spot is fairly uniform over the scale of the optical resolution ( $c a .500 \mathrm{~nm}$ ). The relation to morphological structures is hence clearly established. In the second Raman image [Fig. 6(C)] the lens tissue is imaged at a Raman shift of $1004 \mathrm{~cm}^{-1}$, coincident with the vibrational mode in phenylalanine. The cataractous spot is dark against a light background of the high-intensity Raman signal. The direct conclusion results that the cataractous spot contains smaller amounts of phenylalanine. The total amount of protein is, however, not different.

\section{Cobalt octacarboxyphthalocyanine in single living cells}

In photodynamic therapy, photosensitive molecules are selectively accumulated in tumor tissue. Subsequent irradiation of the tissue with light leads to the formation of compounds that are harmful to the cell. Phthalocyanins are used. Many phthalocyanins with aluminum or zinc as a central atom, have a high fluorescence quantum yield. The distribution of phthalocyanins in the cell and their accumulation in cellular organelles can be investigated with fluorescence imaging methods. Cobalt octacarboxyphthalocyanine is a compound with a low fluorescence quantum yield and Raman imaging is therefore the method of choice. Raman imaging is strongly aided by the high (resonant) Raman crosssection of these compounds when excited with red laser light. Hydrophilic phthalocyanines accumulate in cells mainly by endocytosis. It must therefore be expected that particularly in the lysosomes these compounds can be retrieved. The Raman image lends some support to this view. The bright spots observed in the Raman image in Fig. 8 are with high probability lysosomes. It is also clear from this picture that phthalocyanins are very locally present in the cell. The less bright part of the image could well be out of focus contribution of lower (or higher) lying lysosomes. Preliminary information from scans along the optical axis confirm this idea. It is estimated that $c a .5000$ phthalocyanine molecules in the measurement volume of $c a .1 \mu^{3}$ can be observed. No fluorescent image of cobalt octacarboxyphthalocyanins can be made at this concentration.

\section{Acknowledgements}

We thank Professor Dr C. A. van Blitterswijk of Leiden University for providing us with bone implants. We are grateful to Dr A. Yu. Chikishev of Moscow State University who generously provided us with cobalt octacarboxyphthalocyanine. We thank Dr E. Pels and coworkers of the Cornea Bank, Amsterdam, and Professor Dr G. F. J. M. Vrensen of the Netherlands Ophthalmic Research Institute for the eye-lenses that were made available to us. The highly skilled technical assistance of Mrs I. Segers-Nolten in the preparation of the samples is gratefully acknowledged.
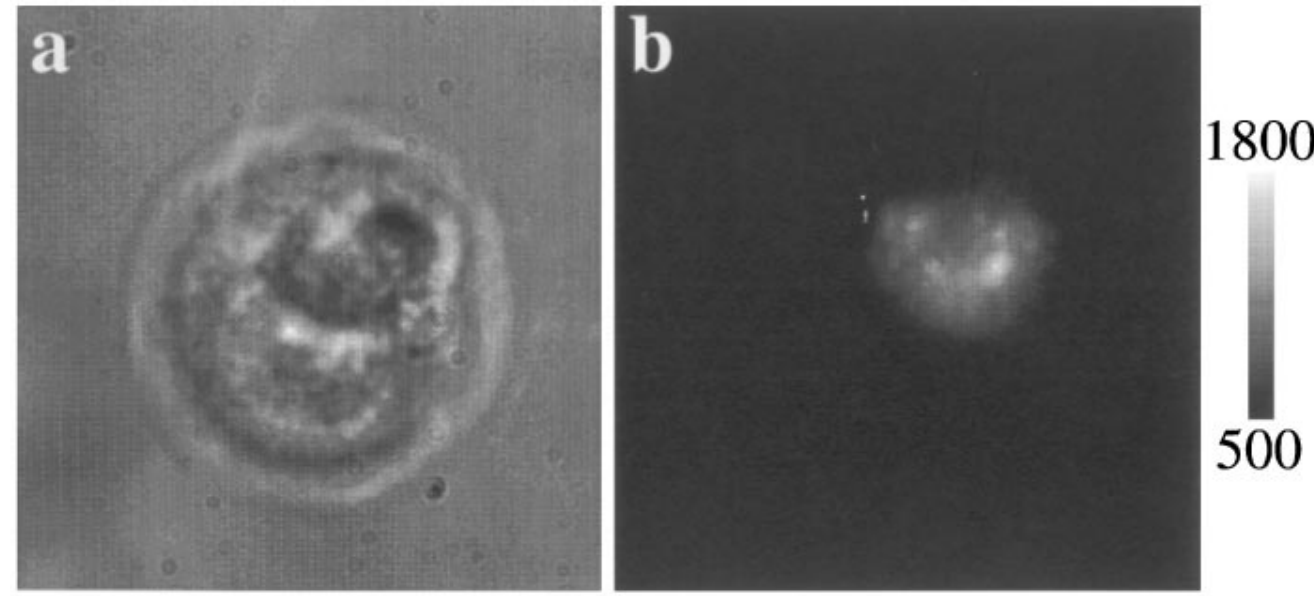

Figure 8. (a) A white light image of a K562 cell. These cells have a large nucleus, the circumference of which roughly allows the cellular surface. A dark region can be recognized in the picture, approximately at two o'clock from the center of the picture. This area contains lysosomal organelles of a granular nature. (b) Raman image of the distribution of cobalt octacarboxyphthalocyanine. The images (a) and (b) can be directly compared. The distribution of cobalt octacarboxyphthalocyanine coincides with the position of lysosomal particles inside the cell. 


\section{REFERENCES}

1. J. Greve and G. J. Puppels, in Advances in Infrared and Raman Spectroscopy, edited by R. J. H. Clark and R. E. Hester, Vol. 20, p. 231. Wiley, Chichester (1993).

2. P. J. Treado and M. D. Morris, Appl. Spectrosc. Rev. 29, 1 (1994).

3. G. J. Puppels, W. Colier, J. H. F. Olminkhof, C. Otto, F. F. M. de Mul and J. Greve, J. Raman Spectrosc. 22, 217 (1991).

4. F. F. M. de Mul, A. G. M. van Welie, C. Otto, J. Mud and J. Greve, J. Raman Spectrosc. 15, 268 (1984).

5. W. L. Kubasek, Y. Wang, G. A. Thomas, T. W. Patapoff, K.-H Schoenwalder, J. H. van der Sande and W. L. Peticolas, Biochemistry 25, 7440 (1986)

6. T. Wilson and A. R. Carlini, Opt. Lett. 12, 227 (1987).

7. G. J. Puppels, F. F. M. de Mul, C. Otto, J. Greve, M. RobertNicoud, D. J. Arndt-Jovin and T. M. Jovin, Nature (London) 347, 301 (1990).

8. G. J. Puppels, C. Otto, J. Greve, M. Robert-Nicoud, D. J Arndt-Jovin and T. M. Jovin, Biochemistry 33, 3386 (1994).

9. G. J. Puppels, J. H. F. Olminkhof, G. M. J. Segers-Nolten, C Otto, F. F. M. de Mul and J. Greve, Exp. Cell Res. 195, 361 (1991).

10. M. Delhaye and P. Dhamelincourt, J. Raman Spectrosc. 3, 33 (1975).
11. J. Barbillat, P. Dhamelincourt, M. Delhaye and E. Da Silva, J. Raman Spectrosc. 25, 3 (1994).

12. S. Sharonov, I. Nabiev, I. Chourpa, A. Feofanov, P. Valisa and M. Manfait, J. Raman Spectrosc. 25, 699 (1994)

13. M. Bowden, D. J. Gardiner, G. Rice and D. L. Gerrard, J. Raman Spectrosc. 21, 37 (1990)

14. D. N. Batchelder, C. Cheng, W. Müller and B. J. E. Smith Makromol. Chem., Macromol. Symp. 46, 171 (1991)

15. G. J. Puppels, M. Grond and J. Greve, Appl. Spectrosc. 47 1256 (1993).

16. M. Robert, Chromosoma 36, 1 (1971)

17. H. J. Duindam, G. F. J. M. Vrensen, C. Otto, G. J. Puppels and J. Greve, J. Lipid Res. 36, 1139 (1995).

18. R. Bittman, Lipids 13, 686 (1978).

19. J. M. Robinson and M. J. Karnovsky, J. Histochem. Cytochem. 28, 161 (1980).

20. J. J. Duindam, G. F. J. M. Vrensen, C. Otto and J. Greve, in preparation.

21. F. F. M. de Mul, M. H. J. Hottenhuis, P. Bouter, J. Greve, J. Arends and J. J. ten Bosch, J. Dent. Res. 65, 437 (1986).

22. G. J. Puppels, PhD Thesis, University of Twente (1991).

23. J. J. Duindam, G. F. J. M. Vrensen, C. Otto and J. Greve, Ophthalmic Res. 28, 86 (1996). 